\section{A circularidade dos médicos em cinco regiões de São Paulo, Brasil: padrões e fatores intervenientes}

\author{
Physicians' commuting in five health regions \\ in São Paulo, Brazil: patterns and intervening \\ factors
}

\section{La circularidad de los médicos en cinco regiones de salud de São Paulo, Brasil: patrones y factores intervinientes}

\section{Resumo}

O artigo objetiva analisar a movimentação dos médicos, sob nova perspectiva, verificando seu deslocamento e oferta de trabalho entre as Regiões de Saúde, especificamente em cinco regiões do Estado de São Paulo, Brasil. Denominouse essa movimentação como circularidade médica, definida pela diversidade de vínculos constituintes do exercício profissional observada ao longo de um determinado período em determinados espaços geográficos. A metodologia usada foi de estudo de casos múltiplos com aplicação de abordagens quantitativas e qualitativas. Todos os médicos cadastrados na base do Cadastro Nacional de Estabelecimentos de Saúde (CNES), em março de 2015, foram categorizados em: "médicos exclusivos", com vínculos exclusivamente na região em foco; $e$ "médicos não exclusivos", com vínculo na região e em outras. Analisaram-se os dados socioeconômicos e de saúde da região e a estrutura assistencial de saúde. A dependência regional de médicos externos variou de $30 \%$ a 40\%, mais elevada nas regiões mais desenvolvidas e menor nas menos desenvolvidas. A dependência interna, entre municípios, fica próxima de $40 \%$ nas regiões com maior desenvolvimento econômico e chega a $60 \%$ nas as regiões menos desenvolvidas. Médicos não exclusivos são mais especializados, com maior atuação em especialidades cirúrgicas e de diagnóstico, e os exclusivos atuam mais em especialidades básicas e clínicas, indicando que a movimentação pode estar associada à organização da prestação da assistência, nos seus diferentes arranjos. Identifica-se uma crescente participação de arranjos terceirizados e a importância de ações pactuadas regionalmente. Tais estudos podem orientar melhor as politicas redistributivas mais integradas.

Regionalização; Recursos Humanos em Saúde; Médicos; Migração Interna; Sistemas de Saúde
Paulo Henrique D'Ângelo Seixas 1,2

Nelson Ibañez 1

Joana Azevedo da Silva 2,3

Ana Cecilia Venci Bueno 2,3

Sabrina Lima 2,3

doi: 10.1590/0102-311X00135018

\section{Correspondência}

P. H. D'A. Seixas

Rua Carlos Weber 499, apto. 11A, São Paulo, SP

05303-000, Brasil.

seixaspaulo@hotmail.com

1 Faculdade de Ciências Médicas da Santa Casa de São Paulo São Paulo, Brasil.

2 Observatório de Recursos Humanos em Saúde de São Paulo, São Paulo, Brasil.

3 Centro de Estudos Augusto Leopoldo Ayrosa Galvão, São Paulo, Brasil. 


\section{Introdução}

A concretização de sistemas de saúde regionalizados e integrados implicou a criação das Redes de Atenção à Saúde (RAS) no Brasil 1, definidas como um arranjo organizativo de ações e serviços, integrado por sistemas de apoio técnico, logístico e de gestão ${ }^{2}$. Tal reorientação apresenta o desafio de conceber e implementar a regionalização e redes como estratégias na mudança do Sistema Único de Saúde (SUS) 3,4,5. Uma variável importante a ser considerada é a desigualdade na oferta de recursos humanos, gerando problemas de acesso. Destacam-se, nesse desequilíbrio, a escassez de médicos em diferentes dimensões - atenção básica, serviços especializados e entre os setores públicos e privado 6 .

Se a má distribuição de recursos humanos no território nacional é, parcialmente, condicionada pelas desigualdades econômicas, as políticas públicas redistributivas voltadas para a força de trabalho em saúde têm-se mostrado pouco resolutivas no enfrentamento deste problema 7,8,9.

Enfoques centrados na dinamicidade dos sistemas de saúde, utilizando diferentes conceitos: migração 10 , rotatividade 11,12, multiplicidade/diversidade de vínculos 13,14, têm sido importantes para entender os movimentos dos profissionais e os fluxos dos pacientes. Os trabalhos referem impactos positivos e negativos dessa mobilidade 15,16 .

Uma breve revisão do tema migração pareceu apropriada à discussão das teorias sobre os processos migratórios, em duas grandes abordagens: micro e macroeconômicas 17,18.

As abordagens microeconômicas estão relacionadas às escolhas individuais e a fatores de atração e repulsão (push-pull) 17, associando-se às oportunidades de trabalho, melhores rendimentos, condições adequadas de trabalho e qualidade de vida.

Os enfoques relacionados ao desenvolvimento profissional 19 e ao ciclo de vida do indivíduo incorporam perspectivas de médio e longo prazos, como oportunidades de crescimento, qualificação, etapas de formação, composição familiar e estabilidade obtida.

Uma abordagem mais integrada 20 relaciona mobilidade espacial à mobilidade profissional e social, constituindo tipologias de migração: empresarial (capital próprio, voltada a novos mercados), organizacional (por meio de organizações em que atua, buscando carreira, promoção) e movimentações individuais direcionadas a novas ocupações ou qualificação.

As abordagens macroeconômicas referem-se a transformações no mercado de trabalho globalizado: segmentação com surgimento de setores informais ou secundários ou, em segmentos formais, o fenômeno de brain drain (saída de pessoas qualificadas de um país, setor econômico ou campo para outro, geralmente por melhores salários ou condições de vida) 21,22. Tais movimentos são voltados para a economia de escala e aglomerações, considerando a disponibilidade de recursos produtivos, criando realidades regionais e urbanas específicas. Alguns autores 23,24 utilizam a chamada Teoria Gravitacional como representação dessa abordagem. Estudos nacionais como o da divisão urbano regional 25 organizam e categorizam as regiões com base nas relações formadas em rede e no comando das cidades. O papel das instituições como agentes empregadores (empresas privadas e públicas, Estado), agenciadores de emprego, associações de apoio a migrantes, são referidos como fatores importantes nesse processo 17 .

Este artigo apresenta a abordagem inicial do conceito de circularidade médica, visando a preencher uma lacuna da literatura na identificação de singularidades desta movimentação no cotidiano de trabalho do profissional, considerando variáveis como espaço territorial, tempo, multiplicidade de vínculos, caracterizando padrões de movimentação regional e como se organizam em termos profissionais e corporativos 26,27 .

Estudos anteriores 26,27 já identificaram alguns padrões dessas movimentações entre serviços, municípios e regiões, respondendo afirmativamente sobre essa circulação (aproximadamente 46,5\% dos médicos atuam em mais de uma Região de Saúde). Na circulação intermunicipal, observa-se a composição de profissionais exclusivos, concentrados nos maiores centros, já os profissionais circulantes atuam em municípios menores, estabelecendo a oferta de médicos para uma região. O estudo atual aprofunda a descrição anterior propondo análise das características e formas de organização dos profissionais, as especialidades envolvidas, bem como a estrutura socioeconômica e assistencial e eventuais arranjos institucionais relacionados à movimentação. 
Nesse sentido, o objetivo central deste artigo é analisar, mediante o conceito da circularidade médica regional, a movimentação dos médicos e sua oferta de trabalho, em cinco regiões do Estado de São Paulo, identificando padrões singulares e fatores relacionados, visando a trazer elementos para as políticas públicas redistributivas.

\section{Método}

Para este estudo foram selecionadas cinco regiões pelo projeto Saúde em Ação (http://www.saudee macao.saude.sp.gov.br), da Secretaria da Saúde do Estado de São Paulo, em parceria com o Banco Interamericano de Desenvolvimento (BID), utilizando critérios políticos e técnicos. Para o escopo da pesquisa, procurou-se enquadrar tais escolhas adotando-se critérios do Estudo Regiões e Redes 3, que classifica as regiões do país em cinco categorias baseadas em indicadores compostos, socioeconômicos e de saúde. O gradiente parte do nível 1, caracterizado por baixo desenvolvimento econômico e baixa oferta de serviços, até o nível 5, com alto desenvolvimento econômico e alta oferta de serviços. Para maior detalhamento das regiões, foram usados outros indicadores: população regional, renda familiar per capita regional, variação do Índice de Desenvolvimento Humano Municipal (IDH-M), cobertura populacional estimada pelas equipes de atenção básica e cobertura populacional por planos de saúde.

As regiões selecionadas apresentaram indicadores variando em gradiente de situações mais vulneráveis, pertencentes à franja oeste do estado - Vale do Ribeira, Vale do Jurumirim, Itapeva -, uma região intermediária, Litoral Norte e a Região Metropolitana de Campinas, com menos vulnerabilidade.

Optou-se por realizar um estudo de casos múltiplos 28 , pela variabilidade de contextos observada nessas regiões. Os procedimentos metodológicos utilizaram abordagens quantitativas e qualitativas: entrevistas com gestores e profissionais médicos, análise documental e banco de dados secundários criado com indicadores dessas regiões. Procedeu-se, assim, a uma triangulação de dados 29, visando a abranger a máxima amplitude na descrição e compreensão do foco do estudo, com cruzamento de múltiplas perspectivas, com base em vários informantes, e diferentes instrumentos de coleta de dados.

A metodologia qualitativa baseou-se na aplicação de roteiros semiestruturados, específicos por categoria, com profissionais médicos e gestores. As entrevistas foram realizadas nos serviços de atuação dos profissionais, gravadas, transcritas e analisadas mediante a metodologia de análise de conteúdo 30. Foi feita pré-análise das entrevistas e a exploração das mesmas, propiciando a criação de unidades de análise face aos objetivos propostos. Baseando-se nessa sistematização, foram criadas categorias de análise e procedeu-se o tratamento e a interpretação dos resultados.

$\mathrm{O}$ roteiro aplicado aos profissionais médicos abordava dados pessoais e profissionais; trajetória profissional/movimentação; vínculos empregatícios/remuneração; motivações e estratégias para atuação na região; protocolos e atualizações. Aos gestores, o roteiro solicitou problemas e estratégias para a atração de profissionais, dificuldades de captação de especialistas e formas de contratação utilizadas/preferidas. Foram entrevistados 114 médicos: 49 da Região Metropolitana de Campinas; 19 do Litoral Norte; 13 de Itapeva; 13 do Vale do Jurumirim; e 20 do Vale do Ribeira. Foram entrevistados 60 gestores, das secretarias municipais, das Regiões de Saúde e de 30 serviços.

A identificação dos médicos foi feita por meio do Cadastro Nacional de Estabelecimentos de Saúde (CNES). O CNES, do Ministério da Saúde 31, determina que todos os estabelecimentos que prestem assistência à saúde, públicos e privados, em todo território nacional, devem ser cadastrados e mantidos atualizados os seus bancos de dados, nas bases locais e federal.

A seleção dos médicos foi feita valendo-se da amostra de conveniência baseada nos critérios: diversidade de inserção (serviços de baixa, média e alta complexidades), idade (diferentes faixas etárias), formação (profissionais com e sem especialização formal), mobilidade (profissionais exclusivos e não exclusivos) e diversidade de vínculos. Os serviços foram escolhidos em função das redes priorizados pela pesquisa, principalmente aqueles de referência para média e alta complexidades. A discussão direcionada às tipologias de circulação representa um recorte da análise qualitativa realizada, seguindo os objetivos deste artigo.

Para a análise quantitativa foram identificados, na competência de março de 2015 , todos os profissionais médicos cadastrados no CNES, categorizados em: "exclusivos", com vínculos exclusivamente na região e "não exclusivos", com vínculo na região e em outras. Foi possível identificar, nas regiões, 
padrões de circulação intra e inter-regionais, sua dependência de trabalhos externos e sua capacidade de retenção.

Os dados foram filtrados pela Classificação Brasileira de Ocupações (CBO) ${ }^{32}$, selecionadas todas as ocupações médicas e categorizadas em grandes áreas: clínica geral, cirurgia geral, pediatria, gineco-obstetrícia, especialidades clínicas, especialidades cirúrgicas, especialidades de apoio diagnóstico e terapêutico 26,27. Para os cálculos envolvendo densidades demográficas, usaram-se as estimativas de população residente nos munícipios com referência para março de 2015, obtidas do banco de dados do Instituto Brasileiro de Geografia e Estatística (IBGE).

Em relação à distribuição dos médicos, foram utilizados indicadores: número de médicos, médicos/mil habitantes, percentual de vínculos com o SUS, idade média, sexo, percentual de graduados imigrantes. Consideraram-se imigrantes aqueles profissionais graduados fora do estado de destino (São Paulo), mas com registro no Conselho Regional de Medicina de São Paulo (Cremesp).

O estudo da circularidade utilizou os seguintes indicadores para a comparação entre os profissionais exclusivos e não exclusivos: número de vínculos na região e externos; especialização (obtida mediante a realização da residência médica credenciada pelo Ministério da Educação ou do título de especialista por meio de prova realizada pela Sociedade de Especialistas, vinculada à Associação Médica Brasileira) e área de atuação (CBO-CNES); frequência de atuação em serviços, cidades, regiões de saúde. Os dados de local de graduação, residência médica e titulação de especialistas foram obtidos por intermédio do Cadastro Nacional de Especialistas cedido pelo Ministério da Saúde.

O tratamento e a análise dos dados foram feitos com o uso do ambiente de programação R versão 3.1.1 (http://www.r-project.org), curados de forma semiautomática e verificados manualmente quanto à consistência e integridade.

A pesquisa foi aprovada pelo Comitê de Ética em Pesquisa em Seres Humanos da Irmandade da Santa Casa de Misericórdia de São Paulo, com o número do parecer: 2.020.191. Conforme Resoluções no 196/96 e no 466/12, sendo considerado risco mínimo, não necessitando seu envio para a Comissão Nacional de Ética em Pesquisa. Contou com Termo de Consentimento Livre e Esclarecido e demais exigências da Resolução CNS no 196/96.

\section{Resultados}

\section{Caracterização das regiões}

Os dados populacionais apresentados (Região Metropolitana de Campinas 3.094.181 habitantes, e demais regiões com média inferior a 300 mil habitantes) apontam para as questões de escala e concentração de estruturas e fluxos 24 . Pelas definições do IBGE 33, a Região Metropolitana de Campinas é a única classificada como centro sub-regional de "Articulação Urbana Intermediária" das 161 áreas no país. As demais regiões, de "Articulação Urbana Imediata” em 482 áreas, classificam-se como centros sub-regionais menores ou centros de zona. Dentro da tipologia das regiões - segundo condições socioeconômicas e de saúde 3 - elas estão no gradiente de 5 a 2 . As regiões classificadas entre 2 e 4 apresentam serviços de média e baixa complexidades 34 . Os indicadores de alta cobertura populacional pelas equipes de atenção básica reforçam a baixa complexidade dos serviços ofertados e sua capilaridade municipal nas regiões como um dos condicionantes da mobilidade médica (Tabela 1).

Em relação aos indicadores Produto Interno Bruto (PIB), renda familiar per capita e IDH, o continuum de resultados encontrados corrobora a análise dos dados apresentados, sendo mais elevados na Região Metropolitana de Campinas. O Litoral Norte conta com um PIB per capita alto explicado pelos royalties do petróleo, além do turismo.

O indicador variação do IDH-M demonstra as desigualdades regionais, evidenciando concentração de melhores condições entre os chamados municípios-polo frente aos demais.

O indicador cobertura populacional por planos de saúde mostra elevada taxa na Região Metropolitana de Campinas, 67\%, já no Litoral Norte é de 26,7\% e 12,7\% no Vale do Ribeira e Itapeva, sugerindo que as opções de mobilidade para os médicos nestas regiões estejam relacionadas aos serviços ofertados pelo SUS. 


\section{Tabela 1}

Características das regiões do estudo e do Estado de São Paulo, Brasil, 2015.

\begin{tabular}{|c|c|c|c|c|c|c|}
\hline Características & $\begin{array}{c}\text { Região } \\
\text { Metropolitana } \\
\text { de Campinas }\end{array}$ & Litoral Norte & $\begin{array}{c}\text { Vale do } \\
\text { Jurumirim }\end{array}$ & Vale do Ribeira & Itapeva & $\begin{array}{c}\text { Estado de São } \\
\text { Paulo }\end{array}$ \\
\hline População estimada (2015) & 3.094 .181 & 308.843 & 295.957 & 284.469 & 282.564 & 44.396 .484 \\
\hline $\begin{array}{l}\text { Tipologia das regiões - } \\
\text { segundo condições de } \\
\text { socioeconômicas de saúde * }\end{array}$ & 5 & 4 & 3 & 2 & 2 & - \\
\hline PIB per capita regional & 47.174 & 42.287 & 18.454 & 30.994 & 17.814 & 39.122 \\
\hline $\begin{array}{l}\text { Renda familiar per capita } \\
\text { regional de } 2010 \text { (valor } \\
\text { máximo e mínimo dos } \\
\text { municípios da região) }\end{array}$ & $\begin{array}{c}947 \\
(1351-569)\end{array}$ & $\begin{array}{c}668 \\
(713-594)\end{array}$ & $\begin{array}{c}598 \\
(753-409)\end{array}$ & $\begin{array}{c}454 \\
(601-352)\end{array}$ & $\begin{array}{c}429 \\
(532-324)\end{array}$ & $\begin{array}{c}853 \\
(1.613-289)\end{array}$ \\
\hline Variação de IDH-M na região & $\begin{array}{c}\text { Muito alto: } 0,819 \\
\text { Alto: } 0,702\end{array}$ & $\begin{array}{l}\text { Alto: } 0,772 \\
\text { Alto: } 0,751\end{array}$ & $\begin{array}{c}\text { Alto: 0,767 } \\
\text { Médio: 0,668 }\end{array}$ & $\begin{array}{c}\text { Alto: 0,754 } \\
\text { Médio: 0,641 }\end{array}$ & $\begin{array}{c}\text { Alto: 0,732 } \\
\text { Médio: 0,642 }\end{array}$ & $\begin{array}{c}\text { Muito alto: 0,862 } \\
\text { Médio: 0,639 }\end{array}$ \\
\hline $\begin{array}{l}\text { Cobertura populacional } \\
\text { estimada pelas equipes de } \\
\text { atenção básica em } 2015 \text { (\%) }\end{array}$ & 73,4 & 86,1 & 77,8 & 93,0 & 78,6 & 63,5 \\
\hline $\begin{array}{l}\text { Cobertura populacional } \\
\text { por planos de saúde em } \\
\text { setembro de } 2015(\%)\end{array}$ & 67,8 & 26,7 & 16,4 & 12,7 & 12,7 & 57,9 \\
\hline
\end{tabular}

IDH-M: Índice de Desenvolvimento Humano Municipal.

Fontes: Estudo Gestão Regional e Redes (http://www.gestaoregional.saude.sp.gov.br/sobre-a-pesquisa/, acessado em Mar/2015); Cadastro Nacional de Estabelecimentos de Saúde (CNES. http://www.cnes.saude.gov.br, acessado em Mar/2015) e Conselho Federal de Medicina (CFM. http://www.portal. cfm.org.br, acessado em Mar/2015).

* De acordo com Viana Ald'A et al. 3.

\section{Oferta de profissionais, migração, especialização médica e estrutura assistencial}

A observação da Tabela 2 permite identificar três grupos: a Região Metropolitana de Campinas, com maior oferta de profissionais (número e taxa/habitante), maior participação de profissionais do sexo feminino, menor idade média, menor vinculação dos profissionais ao SUS e menor participação de imigrantes; as regiões da fronteira com o Paraná (Vale do Jurumirim, Itapeva e Vale do Ribeira), com mais baixa oferta, apresentam alta vinculação de profissionais ao SUS, menor participação feminina, idade média em torno de 50 anos e elevada participação de imigrantes. O Litoral Norte registra uma situação intermediária: maior participação feminina e o dobro da oferta de profissionais que o observado nas regiões de fronteira com o Paraná, porém elevada participação de imigrantes.

Os médicos não exclusivos são mais especializados do que aqueles que atuam exclusivamente dentro da região.

Nas duas categorias (médicos exclusivos e não exclusivos), a Região Metropolitana de Campinas apresenta indicadores mais elevados de especialização. A Região do Vale do Ribeira registra baixa especialização em ambas, mas, ao contrário do observado na oferta de profissionais, as regiões do Vale do Jurumirim e de Itapeva apresentam maior especialização formal quando comparadas ao Litoral Norte.

Os vínculos profissionais estão mais concentrados nos hospitais, variando de 55\% na Região Metropolitana de Campinas a 34\% no Vale do Ribeira (Tabela 3). A atuação nas unidades básicas de saúde (UBS) é baixa na Região Metropolitana de Campinas, cerca de 10\%, 20\% no Litoral Norte e Itapeva, e próxima a 30\% no Vale do Jurumirim e Vale do Ribeira. A participação em serviços especializados alcança 15 a 20\% na Região Metropolitana de Campinas, Litoral Norte e Itapeva, relacionados à presença dos Ambulatórios Médicos de Especialidade (AME) nas duas últimas regiões, sendo menor 


\section{Tabela 2}

Indicadores de demografia médica segundo número, relação médico/habitante, vínculo no Sistema Único de Saúde (SUS), sexo, imigração e idade média por regiões. São Paulo, Brasil, 2015.

\begin{tabular}{|c|c|c|c|c|c|c|}
\hline Regiões & $\begin{array}{l}\text { Número de } \\
\text { médicos }\end{array}$ & $\begin{array}{c}\text { Médicos/1.000 } \\
\text { habitantes }\end{array}$ & $\begin{array}{l}\text { Médicos do } \\
\text { SUS (\%) }\end{array}$ & $\begin{array}{c}\text { Sexo feminino } \\
(\%)\end{array}$ & $\begin{array}{c}\text { Graduados } \\
\text { imigrantes (\%) }\end{array}$ & $\begin{array}{c}\text { Idade média } \\
\text { (anos) }\end{array}$ \\
\hline Região Metropolitana de & 9.673 & 3,44 & 73,46 & 44,42 & 36,47 & 46,69 \\
\hline \multicolumn{7}{|l|}{ Campinas } \\
\hline Litoral Norte & 537 & 1,91 & 89,76 & 35,74 & 57,17 & 50,12 \\
\hline Vale do Jurumirim & 329 & 1,19 & 94,83 & 30,00 & 48,63 & 49,97 \\
\hline Itapeva & 305 & 0,93 & 90,50 & 31,14 & 59,14 & 49,60 \\
\hline Vale do Ribeira & 348 & 1,23 & 88,20 & 31,84 & 65,49 & 50,89 \\
\hline
\end{tabular}

Fontes: Estudo Gestão Regional e Redes (http://www.gestaoregional.saude.sp.gov.br/sobre-a-pesquisa/, acessado em Mar/2015); Cadastro Nacional de Estabelecimentos de Saúde (CNES. http://www.cnes.saude.gov.br, acessado em Mar/2015) e Conselho Federal de Medicina (CFM. http://www.portal. cfm.org.br, acessado em Mar/2015).

\section{Tabela 3}

Porcentagem de especialização médica segundo categorias exclusivos e não exclusivos, locais de prática e regiões estudadas do Estado de São Paulo, Brasil, 2015

\begin{tabular}{|c|c|c|c|c|c|c|}
\hline \multirow[t]{2}{*}{ Regiões } & \multicolumn{2}{|c|}{ Especialização médica (\%) } & \multicolumn{4}{|c|}{ Locais de prática (\%) } \\
\hline & Exclusivos & Não exclusivos & Hospital & $\begin{array}{l}\text { Consultório } \\
\text { médico }\end{array}$ & $\begin{array}{c}\text { Unididades } \\
\text { especializadas }\end{array}$ & UBS \\
\hline $\begin{array}{l}\text { Região Metropolitana de } \\
\text { Campinas }\end{array}$ & 56,03 & 68,93 & 55,03 & 15,32 & 16,33 & 8,90 \\
\hline Litoral Norte & 34,07 & 50,00 & 44,77 & 13,32 & 15,21 & 18,70 \\
\hline Vale do Jurumirim & 40,19 & 58,26 & 44,22 & 18,00 & 5,41 & 28,45 \\
\hline Itapeva & 46,23 & 55,94 & 43,83 & 12,74 & 20,75 & 18,32 \\
\hline Vale do Ribeira & 38,14 & 36,73 & 34,06 & 11,12 & 12,00 & 28,92 \\
\hline
\end{tabular}

UBS: unidades básicas de saúde.

Fontes: Estudo Gestão Regional e Redes (http://www.gestaoregional.saude.sp.gov.br/sobre-a-pesquisa/, acessado em Mar/2015); Cadastro Nacional de Estabelecimentos de Saúde (CNES. http://www.cnes.saude.gov.br, acessado em Mar/2015) e Conselho Federal de Medicina (CFM. http://www.portal. cfm.org.br, acessado em Mar/2015).

no Vale do Ribeira e Vale do Jurumirim. Esse último conta com maior presença de vínculos em consultórios individuais, apontando a possibilidade de um setor privado não conveniado nessa região (18\%).

\section{A circularidade dos médicos}

A análise dos indicadores de circularidade, considerando a dependência de profissionais externos e a movimentação dos médicos entre as regiões, mostra maior estabilidade que a análise comparativa dos padrões de oferta.

A dependência inter-regional de profissionais atuando em outras regiões varia entre 31\% (Região Metropolitana de Campinas) e 40\%, sendo mais elevada nas regiões de Itapeva e Vale do Jurumirim (Tabela 4). 


\section{Tabela 4}

Concentração de médicos nos municípios-polo, índice de dependência regional e intermunicipal, vínculo "não exclusivo" e total de vínculos exercidos nas Regiões de Saúde. Estado de São Paulo, Brasil, 2015.

\begin{tabular}{|c|c|c|c|c|c|}
\hline Regiões & $\begin{array}{l}\text { Concentração de } \\
\text { médicos em municípios- } \\
\text { polo }\end{array}$ & $\begin{array}{l}\text { Dependência } \\
\text { regional * }(\%)\end{array}$ & $\begin{array}{c}\text { Dependência } \\
\text { intermunicipal ** } \\
(\%)\end{array}$ & $\begin{array}{l}\text { Vínculos não } \\
\text { exclusivos na } \\
\text { região (\%) }\end{array}$ & $\begin{array}{l}\text { Percentual do total de } \\
\text { vínculos de não exclusivos } \\
\text { exercidos na região }\end{array}$ \\
\hline $\begin{array}{l}\text { Região Metropolitana } \\
\text { de Campinas }\end{array}$ & Campinas: $60 \%$ & 31,08 & 42,89 & 26,80 & 45,27 \\
\hline Litoral Norte & $\begin{array}{l}\text { Caraguatatuba: 35,42\% } \\
\text { São Sebastião: 35,03\% }\end{array}$ & 33,00 & 39,94 & 30,51 & 51,50 \\
\hline Vale do Jurumirim & Avaré: 41,23\% & 35,00 & 61,94 & 27,61 & 51,81 \\
\hline Itapeva & Itapeva: 50,47\% & 40,67 & 54,58 & 28,13 & 41,45 \\
\hline Vale do Ribeira & $\begin{array}{c}\text { Pariquera-Açu: } 38,82 \% \\
\text { Registro: } 38,36 \%\end{array}$ & 33,56 & 66,33 & 26,25 & 29,07 \\
\hline
\end{tabular}

Fonte: Cadastro Nacional de Estabelecimentos de Saúde (CNES. http://www.cnes.saude.gov.br, acessado em Mar/2015).

* Dependência regional: profissionais não exclusivos na região/total de médicos;

** Dependência intermunicipal: profissionais exclusivos na região e não exclusivos no município/total de médicos exclusivos na região.

Na dependência intrarregional (média das dependências de cada um dos municípios da região em relação aos profissionais não exclusivos naquele município, que atuam dentro da região), as diferenças são mais definidas. Variam de 40\% no Litoral Norte e na Região Metropolitana de Campinas até cerca de $60 \%$ nas regiões fronteiriças com o Paraná.

A média de vínculos médicos dentro das regiões situou-se em torno de 2,5, mais elevada para os profissionais com atuação exclusiva. Entretanto, os profissionais não exclusivos têm sua média de vínculos totais variando de 4 a 5, mais elevada nas regiões fronteiriças com o Paraná (Tabela 5).

Os padrões se repetem em relação às atuações em cidades e nos serviços. Os médicos não exclusivos apresentam menor participação do que os exclusivos, no interior das regiões, mas apresentam quase o dobro de atividades totais.

Algumas regiões detêm cerca de 50\% dos vínculos de seus profissionais não exclusivos (Região Metropolitana de Campinas, Litoral Norte e Vale do Jurumirim). Aquelas com maior vulnerabilidade recebem entre 30\% e 40\% dos vínculos de seus profissionais não exclusivos. Considerando que os profissionais sempre atuam em mais de duas regiões $(2,27$ a 2,58), as primeiras parecem ser áreas prioritárias de trabalho e as últimas podem representar áreas de atuação complementares.

Os profissionais não exclusivos apresentam maior especialização formal. Já a distribuição da atuação dos profissionais por grandes grupos de especialidades demonstra distinções relevantes. Os médicos não exclusivos apresentam maior concentração relativa em especialidades cirúrgicas ou em especialidades de apoio diagnóstico. Atuam em serviços mais especializados, com maior tecnologia incorporada. Os profissionais exclusivos, comparativamente, atuam mais em clínica médica, especialidades clínicas, ginecologia e obstetrícia e pediatria 35 .

Baseando-se nas análises das entrevistas, percebe-se que a circulação pode acontecer mediante diferentes combinações, relacionadas com as especialidades e estruturas assistenciais. Já foi estudada, em outros momentos, a migração associada à residência médica e seu papel como fator de estabilização nos fluxos desses profissionais no país 36,37. Essa também é identificada como umas das tipologias de migração médica na Europa 16.

Para caracterizar as tipologias de movimentação utilizadas pelos médicos nesses movimentos cotidianos e recorrentes de trabalho, inter-regionais ou intermunicipais, buscou-se estudar iniciativas relacionadas diretamente à inserção do profissional no trabalho. Foram consideradas suas estratégias de oferta de força de trabalho, a associação com outros agentes e a vinculação com o contratante. Identificaram-se três grandes arranjos: o primeiro deles, predominante e bastante pulverizado. Trata-se da movimentação isolada e individual do profissional, buscando, mediante suas redes de relacionamento e seus interesses, oferecer seus serviços como pessoa física ou jurídica aos contratantes. 


\section{Tabela 5}

Valor médio de vínculos, estabelecimentos, cidades, regiões de atuação e especialidades praticadas por médicos exclusivos e não exclusivos em Regiões de Saúde. São Paulo, Brasil, 2015

\begin{tabular}{|c|c|c|c|c|c|c|c|c|c|c|c|c|c|}
\hline \multirow[t]{2}{*}{ Regiões } & \multicolumn{2}{|c|}{$\begin{array}{l}\text { Média de } \\
\text { vínculos/região }\end{array}$} & \multirow{2}{*}{$\begin{array}{l}\text { Média de } \\
\text { vínculos } \\
\text { Totais } \\
\text { não } \\
\text { exclu- } \\
\text { sivos }\end{array}$} & \multicolumn{3}{|c|}{ Estabelecimentos } & \multirow[b]{2}{*}{$\begin{array}{l}\text { Não } \\
\text { exclu- } \\
\text { sivos }\end{array}$} & \multicolumn{2}{|c|}{ Cidades } & \multirow{2}{*}{$\begin{array}{c}\text { Regiões } \\
\text { Não } \\
\text { exclu- } \\
\text { sivos }\end{array}$} & \multicolumn{3}{|c|}{$\begin{array}{c}\text { Número de especialidades } \\
\text { referidas no CNES }\end{array}$} \\
\hline & $\begin{array}{c}\text { Não } \\
\text { exclu- } \\
\text { sivos }\end{array}$ & $\begin{array}{l}\text { Exclu- } \\
\text { sivos }\end{array}$ & & $\begin{array}{l}\text { Não } \\
\text { exclu- } \\
\text { sivos }\end{array}$ & $\begin{array}{l}\text { Exclu- } \\
\text { sivos }\end{array}$ & $\begin{array}{c}\text { Totais não } \\
\text { exclu- } \\
\text { sivos * }\end{array}$ & & $\begin{array}{l}\text { Exclu- } \\
\text { sivos }\end{array}$ & $\begin{array}{c}\text { Totais não } \\
\text { exclu- } \\
\text { sivos ** }\end{array}$ & & $\begin{array}{c}\text { Não } \\
\text { exclu- } \\
\text { sivos }\end{array}$ & $\begin{array}{l}\text { Exclu- } \\
\text { sivos }\end{array}$ & $\begin{array}{c}\text { Totais } \\
\text { não } \\
\text { exclu- } \\
\text { sivos *** }\end{array}$ \\
\hline $\begin{array}{l}\text { Região } \\
\text { Metropolitana } \\
\text { de Campinas }\end{array}$ & 2,01 & 2,52 & 4,44 & 1,85 & 2,34 & 3,84 & 1,28 & 1,34 & 2,72 & 2,3 & 1,28 & 1,36 & 1,96 \\
\hline Litoral Norte & 2,19 & 2,43 & 4,25 & 1,84 & 2,06 & 3,66 & 1,24 & 1,24 & 2,64 & 2,27 & 1,39 & 1,45 & 1,90 \\
\hline $\begin{array}{l}\text { Vale do } \\
\text { Jurumirim }\end{array}$ & 2,57 & 3,63 & 4,96 & 2,15 & 2,7 & 4,17 & 1,37 & 1,45 & 2,92 & 2,31 & 1,58 & 2,06 & 2,09 \\
\hline Itapeva & 2,06 & 3,61 & 4,97 & 1,72 & 2,85 & 4,30 & 1,15 & 1,29 & 2,90 & 2,48 & 1,41 & 2,02 & 2,12 \\
\hline $\begin{array}{l}\text { Vale do } \\
\text { Ribeira }\end{array}$ & 1,39 & 1,97 & 4,78 & 1,35 & 1,78 & 4,57 & 1,23 & 1,42 & 3,37 & 2,58 & 1,63 & 1,29 & 2,20 \\
\hline
\end{tabular}

CNES: Cadastro Nacional de Estabelecimentos de Saúde.

Fonte: Cadastro Nacional de Estabelecimentos de Saúde (CNES. http://www.cnes.saude.gov.br, acessado em Mar/2015).

* Total de estabelecimentos dos profissionais não exclusivos incluindo aqueles fora da região;

** Total de cidades dos profissionais não exclusivos incluindo aquelas fora da região;

*** Total de especialidades dos profissionais não exclusivos incluindo aquelas fora da região.

O segundo perfil está associado a pequenos grupos ou empresas médicas. São mais organizados em torno de especialidades, contratados para operar determinados setores hospitalares - ortopedia, anestesia, pediatria, UTI, obstetrícia, Serviço de Apoio Diagnóstico Terapêutico (SADT).

Um terceiro perfil, organizacional, é associado a empresas empregadoras atuando em diferentes sedes, ou a empresas de gerenciamento de serviços como Organizações Sociais de Saúde (OSS), com capacidade de captação e deslocamento de profissionais em diferentes regiões. Essas, eventualmente atuam com um raio de ação maior.

\section{Discussão}

O perfil socioeconômico das regiões e sua estrutura assistencial têm grande poder explicativo quando relacionados à oferta e distribuição dos profissionais, bem como aos movimentos migratórios e à especialização médica.

Considerando os fatores neoclássicos (push-pull) 17,18, diversas revisões apontam para a importância das condições de trabalho e remuneração, possibilidade de desenvolvimento profissional, qualificação, bem como volume de atendimento, oferta diversificada de trabalho, e estrutura diagnóstica como componentes importantes da fixação do profissional 36,37,38. Compõem esse pacote fatores como qualidade de vida, segurança e lazer, emprego para o cônjuge e oferta de educacional adequada para os filhos 37,38 .

A Organização Mundial da Saúde (OMS) apresenta quatro campos de intervenção para as políticas redistributivas - educacionais, profissionais, regulatórias e de suporte pessoal 36. Entretanto, além de identificar o que atrai, é importante definir quando e como utilizar tais estratégias. Os autores recomendam políticas mais abrangentes e integradas e a adaptação às conjunturas locais, considerando os arranjos institucionais existentes.

A Região Metropolitana de Campinas (tipo 5) ${ }^{3}$ é considerada quase como um modelo para a atração e fixação de profissionais de saúde. O fator escala tem importante peso, envolvendo a densidade 
de centros de formação e especialização médica ${ }^{39}$. Seus profissionais estão mais concentrados em atividades hospitalares, serviços especializados e em consultórios particulares, compatível com uma região com serviços de alta complexidade, alta cobertura populacional por planos de saúde (população com capacidade de consumo e setor formal da economia bem estruturado) e elevada qualidade de vida.

No Litoral Norte (tipo 4), os aspectos macroeconômicos, a concentração de população em apenas quatro municípios e a qualidade de vida na região litorânea justificam a boa oferta de profissionais, como também a razoável estrutura assistencial dos municípios, constituída por uma rede básica com boa cobertura e rede hospitalar pública de média complexidade. A elevada participação de imigrantes e a baixa especialização médica podem estar associadas à expansão dos serviços direcionados à atenção básica, aos serviços de urgência e emergência e Unidades de Pronto Atendimento em Saúde (UPA), envolvendo profissionais menos especializados. Essa rede apresenta menor atratividade no setor privado (27\% cobertura de saúde suplementar) devido à informalidade do setor produtivo local, considerando a sazonalidade de região turística litorânea. Região fronteiriça com o Rio de Janeiro, conta com a participação de médicos imigrantes jovens, transitórios, associados a um grupo de médicos moradores de mais idade. Entretanto, apresenta menor atratividade para fixação de profissionais do próprio estado.

As regiões de fronteira com o Paraná apresentam os fatores invertidos de atração e repulsão em relação à Região Metropolitana de Campinas, com particularidades institucionais específicas.

No Vale do Ribeira (grupo 2), identifica-se ausência de escala, menor desenvolvimento econômico, baixa complexidade de serviços, ausência de setor formador, população dispersa e dificuldades de acesso. Entretanto, o investimento público no complexo Hospital Regional/Ambulatório Médico de Especialidades (AME) consegue oferecer profissionais para esses serviços de maior complexidade. Por outro lado, a dificuldade de integração entre os agentes locais limita a ampliação de oferta para a região. Itapeva (grupo 2), dentro do mesmo padrão, registra a proximidade de um centro formador ao município-polo e a associação OSS da Santa Casa de Itapeva com o AME regional. Essas características ajudam a explicar a maior participação de especialistas na região, também concentrada no município-polo.

A Região do Vale do Jurumirim (grupo 3) apresenta como fatores relevantes a proximidade de centros formadores, estrutura de serviços com presença de Santas Casas locais e flexibilidade de contratação por intermédio de OSS municipal. Qualidade de vida (estância turística), acesso e serviços melhor distribuídos pela região também contribuem para melhorar a oferta regional.

Essas regiões apresentam maior proporção de médicos imigrantes, pois, além de constituírem regiões de fronteira, registram baixa atração para os profissionais do próprio estado. Nesse sentido, os imigrantes cumprem o papel habitual de ocupar postos de trabalho menos procurados pelos profissionais locais 10,16,39. A menor participação feminina nas regiões de fronteira é compatível com a menor disponibilidade para imigração destas profissionais 40,41.

Considerando os mecanismos possíveis para ampliar a oferta do trabalho necessário em uma região 37: importação de profissionais ou ampliação do tempo dedicado daqueles já em atividade, ampliação do escopo de prática 42 de outros profissionais, definição de novas parcerias de contratação, a circularidade contribui significativamente com a primeira e ajuda a compreender a última. Identificar como e por que os profissionais vêm às regiões de maior vulnerabilidade, e analisar os arranjos envolvidos neste movimento, podem auxiliar muito as ações fragmentadas já realizadas pelos agentes públicos.

A análise da circularidade intra e inter-regional tenciona e complementa o estudo sobre oferta regional e migração. A circulação inter-regional, significativa em todas as regiões, é mais intensa nas regiões menos desenvolvidas, sinalizando sua relação com situações de menor oferta médica. A menor disponibilidade de profissionais abre espaço para atuações de profissionais externos, mais especializados que os locais, possibilitando um contrafluxo de profissionais circulantes das áreas mais desenvolvidas, buscando alternativas de trabalho em áreas menos desenvolvidas.

A maior especialização formal dos profissionais não exclusivos é compatível com suas ocupações em serviços com maior incorporação tecnológica. A concentração nas áreas de especialidades cirúrgicas e de apoio diagnóstico aponta para um cuidado ajustado a procedimentos direcionados, específicos e menos dependentes de acompanhamento continuado, e possibilita a atuação do profissional 
em diversos serviços (e regiões), considerando apresentarem praticamente o dobro de vínculos totais dos exclusivos. Esse modelo (investigação diagnóstica, intervenção/orientação de conduta e encaminhamento) é muito associado aos AME regionais que propõem um retorno rápido dos pacientes para a atenção básica - mas também com outros serviços especializados e o trabalho em plantão hospitalar.

Na circularidade, a condição de acesso à região cumpre um papel ainda mais importante. A Região Metropolitana de Campinas, com amplo acesso e atratividade para diferentes centros sub-regionais internos e externos, apresenta uma circulação importante. No sentido oposto, a dificuldade de acesso ao Vale do Ribeira e a oferta muito concentrada no Polo Pariquera-Açu/Registro limitam a circulação local. O mesmo não ocorre com Vale do Jurumirim e Itapeva, que apresentam melhor acesso inter-regional aos municípios-polo.

É possível que a maior dinamicidade dos não exclusivos entre regiões com menor desenvolvimento também esteja relacionada à necessidade de maior movimentação para auferir ganhos que compensem os custos de deslocamento, particularmente nas movimentações inter-regionais.

Já a circulação intrarregional é ainda mais elevada considerando a dependência dos pequenos municípios frente ao município-polo. Aqui, essa circulação não é realizada apenas por intermédio de profissionais mais especializados, mas se aplica também aos profissionais da atenção básica, em prontos-socorros e UPA. A menor oferta de postos de trabalho induz tais profissionais a circularem mais em busca de melhor compensação global.

Esses achados reforçam outros já observados no país 27 e são similares àqueles referidos por alguns autores 43,44 ao tratar de migrações médicas interestaduais.

As tipologias de circulação 27 assemelham-se àquelas propostas por Savage 20: empresarial, organizacional e individual. Entretanto, se naquelas tais movimentos representam uma escolha individual combinada, na circularidade a mediação institucional e transitoriedade manifestam-se de forma bastante intensa.

Grande parte da circulação intrarregional é realizada por meio da tipologia individual. Esse modelo é muito desenvolvido mediante plantões, em áreas de atuação menos especializadas, com menor exigência de qualificação, em serviços de pronto-socorro e realizadas com um raio de deslocamento menor - vários profissionais se utilizam de grupos de WhatsApp para identificar ofertas e valores de plantões regionalmente.

O segundo modelo - empresas/grupos médicos - pode demandar circulações inter-regionais. Em que pese sua enorme plasticidade 45 , implica uma organização mais formal, com um coordenador e estrutura jurídico-administrativa instituída, regras de organização do trabalho, de contribuição, e distribuição de responsabilidades e de rendimento auferido. Pode ser realizada em um raio maior, com o grupo atuando em diversos hospitais.

O terceiro modelo, em que as diretrizes organizacionais e a intenção de evoluir na carreira interna orientam a movimentação, não costumava ser um modelo tão frequente no exercício profissional dos médicos. Mas parece crescer em função da expansão do empresariamento na assistência pública à saúde 46,47 .

As observações referentes às tipologias da circulação médica e seus respectivos arranjos institucionais identificam que esses movimentos são muito relacionados a novos (e antigos) agentes públicos não estatais com diferentes características, presentes em todas as regiões estudadas 48. Os arranjos associados às OSS destacam-se, com modelos característicos: no primeiro, o município ou o estado qualifica um serviço local (Santa Casa) que passa a gerenciar diferentes serviços: AME regional, ambulatório de especialidades, UPA, atenção básica e eventualmente outros hospitais. Em pequenas regiões, a associação Santa Casa-AME, ao constituir oportunidade de trabalho duplicada, tem grande potencial de atração e eventualmente fixação.

Em outro modelo, a OSS estadual, com diferentes locais de atuação e abrangência regional, capta profissionais em municípios-polo e os aloca em outros municípios. Aqui importa ao profissional a garantia do deslocamento, mas também a possibilidade de atuar em múltiplos serviços da organização.

O crescimento de tais modalidades terá consequências importantes para a gestão pública e, mediante as escolhas realizadas, para o poder e para a governança local.

A associação entre o hospital de referência regional e a gerência da referência especializada institui grande poder de negociação de ambos os agentes dentro do sistema local. Já a OSS múltipla passa a ter um enorme significado dentro do sistema, inclusive frente ao próprio agente contratante, reforçando 
a tendência de um modelo de pluralismo empresariado 46, com contratações baseadas na produção, porém, com dificuldades regulatórias e pequena cooperação entre os gestores.

Nesse sentido, faz-se necessário instituir estratégias de negociação e pactuação que possibilitem um maior equilíbrio na identificação de necessidades, na construção do modelo assistencial e no acompanhamento dos resultados em âmbito regional, considerando não apenas a eficiência operacional do serviço, mas sua eficácia e efetividade dentro do sistema.

A estruturação de um sistema mais colaborativo pode contribuir para avanços significativos em termos de ofertas regionais, rompendo com o isolamento dos gestores na contratação de seus profissionais, possibilitando sua distribuição mais adequada na região.

Por fim, é necessário discutir os limites desta pesquisa considerando a metodologia empregada de estudo de casos múltiplos, fixando seus resultados na esfera exploratória sem poder de generalização, apesar de trazer possibilidades de inferências teóricas. Outro limite está nas bases de dados utilizadas. A análise de um mês no CNES pode apresentar limitações na observação dos resultados. Apesar de o CNES ser aplicado à totalidade dos serviços de saúde do país, o registro dos vínculos médicos, principalmente no setor de saúde suplementar, ainda apresenta uma qualidade de informação questionável. O estudo sobre a circularidade identificou e descreveu associações interessantes em termos de movimentação regional, organização do trabalho e institucionalidades, fixando-se mais na análise da tipologia organizacional, dada sua visibilidade estratégica e impacto regional. Cabe ampliar a análise para as demais modalidades, avançando na construção de um referencial mais estruturado, da circularidade em rede, considerando as diferentes formas de organização do trabalho das especialidades médicas e sua relação com esta movimentação, mas também aprofundar estudos frente aos arranjos institucionais envolvidos e seus impactos efetivos, tanto na oferta como nas novas demandas associadas à regulação e à governança regional.

\section{Considerações finais}

Os resultados identificados pelo trabalho mostram a importância de uma compreensão ampliada da circularidade médica entre os serviços de saúde, que considere a dimensão regional para a implantação de políticas públicas do setor.

O primeiro aspecto desta abordagem refere-se ao próprio dimensionamento desta circularidade nos diferentes serviços e regiões. Observa-se que ele é significativo, entre $31 \%$ e $40 \%$ inter-regionalmente, chegando a $60 \%$ internamente. Tais profissionais são fundamentais na oferta de trabalhos intra e inter-regionais.

Importa considerar a variável movimentação e eventualmente, transporte, como componente nas estratégias para a atração dos profissionais, assim como a possibilidade de que estas não se façam apenas com base na perspectiva de fixação, mas que considerem flexibilidade de horários, além de condições de trabalho e remuneração adequadas.

Entretanto, fica muito evidente a fragilidade do agente municipal negociando isoladamente com o conjunto de profissionais que necessita para estruturar seus serviços. Esse conhecimento abre a possibilidade de negociações integradas, com definição de tarefas complementares entre os diferentes serviços e gestores, racionalizando a distribuição do trabalho por toda a região, junto às necessidades de saúde da população.

No caso paulista, um arranjo importante de atração de profissionais acontece por intermédio da implantação dos AME, geridos pelo Estado por meio de OSS, compatíveis com uma ação que integra ação médica especializada, de curto prazo, condições de trabalho adequadas e acesso a serviços diagnósticos. Nessa condição, cabe considerar o papel e o poder que diferentes alianças podem instituir dentro do sistema. Tais arranjos podem ter impactos diferentes na estrutura da prestação local e no próprio poder e capacidade de regulação pública. A análise da circularidade evidencia práticas profissionais e de serviços que podem orientar estratégias mais adequadas e integradas para a contratação e distribuição de profissionais. Entretanto, tais estratégias recolocam mais uma vez a centralidade da estruturação de uma instância regional, constituída por estado e municípios que sejam capazes de identificar necessidades de saúde, comprar e gerir serviços, bem como acompanhar e avaliar de forma adequada o impacto de suas ações sobre o sistema de saúde. 


\section{Colaboradores}

P. H. D’A. Seixas participou da concepção e desenho do estudo, análise e interpretação dos dados, consolidação e redação preliminar do artigo, aprovação conjunta da versão final, responsável pela garantia da exatidão e integridade da obra. N. Ibañez colaborou na revisão crítica do artigo em relação a dimensões abordadas, ajustes de categorias de análise, discussão e aprovação conjunta do texto final e garantia da integridade e exatidão da obra. J. A. Silva contribuiu na análise e interpretação dos dados qualitativos, elaboração de relatório preliminar e apoio à revisão, participação da aprovação conjunta do texto final, compromisso com a exatidão e integridade do trabalho. A. C. V. Bueno contribuiu no detalhamento do referencial para análise e interpretação de dados e elaboração de relatório preliminar do arranjo institucional nas regiões, apoio à revisão do artigo, participação na aprovação do texto final e compromisso com a exatidão e integridade do artigo. S. Lima colaborou na elaboração de referencial para a consolidação e analise de dados secundários, elaboração de relatório preliminar e apoio à revisão integrativa dos casos, participação na aprovação do texto final e garantia à exatidão e integridade da obra.

\section{Informações adicionais}

ORCID: Paulo Henrique D'Angelo Seixas (00000002-8382-2872); Nelson Ibañez (0000-00028459-4736); Joana Azevedo da Silva (0000-00031889-7920); Ana Cecilia Venci Bueno (0000-00018750-1817); Sabrina Lima (0000-0002-5738-6472).

\section{Agradecimentos}

Ao Projeto Fortalecimento à Gestão Estadual em Saúde - SESSP/BID - Estudo Diagnóstico pelo financiamento.

\section{Referências}

1. Guerra DM. Descentralização e regionalização da assistência à saúde no estado de São Paulo: uma análise do índice de dependência [Tese de Doutorado]. São Paulo: Faculdade de Saúde Pública, Universidade de São Paulo; 2015.

2. Ministério da Saúde. Portaria no 4.279, de 30 de dezembro de 2010. Estabelece diretrizes para a organização da Rede de Atenção à Saúde no âmbito do Sistema Único de Saúde (SUS). Diário Oficial da União 2010; $31 \mathrm{dez}$.

3. Viana ALD, Bousquat A, Pereira APCM, Uchimura LYT, Albuquerque MV, Mota PHS, et al. Tipologia das Regiões de Saúde: condicionantes estruturais para a regionalização no Brasil. Saúde Soc 2015; 24:413-22.

4. Viana ALD. Política, planejamento e gestão das regiões e redes de atenção à saúde no Brasil. Resumo executivo. http://www.resbr.net.br/apesquisa/resumo-executivo/ (acessado em 10/ Mar/2018).
5. Fink J. Aligning with physicians to regionalize services. Healthc Financ Manage 2014; 68:80-6.

6. Oliveira APC, Gabriel M, Poz MRD, Dussault G. Desafios para assegurar a disponibilidade e acessibilidade à assistência médica no Sistema Único de Saúde. Ciênc Saúde Colet 2017; 22:1165-80.

7. Secretaria de Políticas da Saúde, Ministério da Saúde. Política de recursos humanos para o SUS: balanço e perspectivas. Brasília: Ministério da Saúde; 2002.

8. World Health Organization. The world health report 2006: working together for health. Geneva: World Health Organization; 2016.

9. Pierantoni CR, Varella TC, Santos MRD, França T, Garcia AC. Gestão do trabalho e da educação em saúde: recursos humanos em duas décadas do SUS. Physis (Rio J.) 2008; 18:685704. 
10. Dussault G, Franceschini MC. Not enough there, too many here: understanding geographical imbalances in the distribution of the health workforce. Hum Resour Health 2006; $4: 12$.

11. Campos CVDA, Malik AM. Satisfação no trabalho e rotatividade dos médicos do Programa Saúde da Família do Município de São Paulo. Rev Adm Pública 2008; 42:347-68

12. Pierantoni CR, Vianna CMM, França T, Magnago C, Rodrigues MPS. Rotatividade da força de trabalho médica no Brasil. Saúde Debate 2015; 39:637-47.

13. Ferrinho P, Biscaia A, Fronteira I, Hipólito F, Dussault G. Multiple employment in health sector in Portugal. Cah Sociol Demogr Med 2007; 47:331-46.

14. Pong RW, Pitblado JR. Geographic distribution of physicians in Canada: beyond how many and where. Ottawa: Canadian Institute for Health Information; 2005.

15. Costigliola V. Mobility of medical doctors in cross-border healthcare. EPMA J 2011;2:333-9.

16. Glinos IA, Buchan J. Health professionals crossing the EU's internal and external borders: a typology of health professional mobility and migration. In: Buchan J, Wisman M, Glinos IA, Bremner J, editors. Health professional mobility in a changing Europe: new dynamics, mobile individuals and diverse responses. v. II. Geneva: European Observatory on Health Systems and Policies/World Health Organization; 2014. p. 129-52. (Observatory Studies Series, 32).

17. Peixoto J. As teorias explicativas das migrações: teorias micro e macro-sociológicas. Lisboa: Centro de Investigação em Sociologia Económica e das Organizações, Instituto Superior de Economia e Gestão, Universidade Técnica de Lisboa; 2004. (Working Papers SOCIUS).

18. Santos MA, Barbieri AF, Carvalho JA, Machado CJ. Migração: uma revisão sobre algumas das principais teorias. Belo Horizonte: Centro de Desenvolvimento e Planejamento Regional, Universidade Federal de Minas Gerais; 2010. (Texto para Discussão, 398).

19. Becker GS. Investment in human capital: a theoretical analysis. J Polit Econ 1962; 70(Part 2) Supplement:9-49.

20. Savage M. The missing link? The relationship between spatial mobility and social mobility. Br J Sociol 1988; 39:554-77.

21. Cambridge English Dictionary. Brain drain. https://dictionary.cambridge.org/us/diction ary/english/brain-drain (acessado em 15/ Mar/2018).

22. Mullan F. The metrics of the physician brain drain. N Engl J Med 2005; 353:1810-8.

23. Lewer JJ, van den Berg H. A gravity model of immigration. Econ Lett 2008; 99:164-7.

24. Barbosa W, Oliveira EA, Freitas CA, Feistel PR. Migrações interestaduais: uma aplicação do modelo gravitacional para os estados brasileiros. Revista Brasileira de Estudos Regionais e Urbanos 2016; 10:156-76.
25. Instituto Brasileiro de Geografia e Estatística. Divisão urbano-regional. Rio de Janeiro: Instituto Brasileiro de Geografia e Estatística; 2013.

26. Seixas PHD, Silvestre DAMM, Viana ALd'A, Uchimura LYT, Pereira APCM. Movimentação médica no Brasil. (Novos Caminhos, 2). http://www.resbr.net.br/wp-content/ uploads/2015/04/MovimentacaoMedica.pdf.

27. Seixas PH, Uchimura LYT, Viana ALD, Silva RC. Circularidade dos médicos nas regiões de saúde no Brasil. Rev Bras Saúde Mater Infant 2017; 17 Suppl 1:S199-208.

28. Yin RK. Estudo de caso: planejamento e métodos. 4a Ed. Porto Alegre: Bookman; 2010.

29. Minayo MCS, Assis SG, Souza ER. Avaliação por triangulação de métodos: abordagem de programas sociais. Rio de Janeiro: Editora Fiocruz; 2005.

30. Bardin L. Análise de conteúdo. Lisboa: Edições 70; 2008.

31. Ministério da Saúde. Portaria MS/SAS 376, de 3 de outubro de 2000. Diário Oficial da União 2000; 4 out.

32. Ministério do Trabalho. Classificação brasileira de ocupações. Brasília: Ministério do Trabalho; 2002.

33. Instituto Brasileiro de Geografia e Estatística. Arranjos populacionais e concentrações urbanas no Brasil. Rio de Janeiro: Instituto Brasileiro de Geografia e Estatística; 2015.

34. Motta DM. Caracterização e tendências da rede urbana do Brasil. v. 1. Rio de Janeiro: Instituto de Pesquisa Econômica Aplicada; 2001. (Série Caracterização e Tendências da Rede Urbana do Brasil).

35. Seixas PHD’A, Silva JA, Bueno ACV, Souza SL. Circulação médica. In: Ibañez N, Vianna AL; Secretaria de Estado da Saúde de São Paulo, organizadores. Estudo diagnóstico regiões e redes. São Paulo: Secretaria de Estado de Sáude de São Paulo/Centro de Estudos Augusto Leopoldo Ayrosa Galvão; no prelo.

36. Dolea C. Increasing access to health workers in remote and rural areas through improved retention: global policy recommendations. Geneva: World Health Organization; 2010.

37. Lehmann U, Dieleman M, Martineau T. Staffing remote rural areas in middle-and lowincome countries: a literature review of attraction and retention. BMC Health Serv Res 2008; 8:19.

38. Buykx P, Humphreys J, Wakerman J, Pashen D. Systematic review of effective retention incentives for health workers in rural and remote areas: towards evidence-based policy. Aust J Rural Health 2010; 18:102-9.

39. Seixas PHD’A, Corrêa A, Moraes JD. Migramed - migração médica no Brasil: tendências e motivações. In: Pierantoni CR, Dal Poz MR, França T, organizadores. O trabalho em saúde: abordagens quantitativas e qualitativas. Rio de Janeiro: Centro de Estudos e Pesquisa em Saúde Coletiva; 2011. p. 133-50. 
40. Seixas PHD'A, Correa AN, Silvestre DDAM, Silveira PSP, Bertolini SR. Migramed II - educação e saúde: condicionantes estruturais e institucionais da atração e fixação de médicos em território nacional. São Paulo: Observatório de Rescursos Humanos em Saúde de São Paulo; 2011.

41. Scheffer MC, Cassenote AJF, Guilloux AGA, Dal Poz MR. Internal migration of physicians who graduated in Brazil between 1980 and 2014. Hum Resour Health 2018; 16:21.

42. Girardi SN, Carvalho CL, Pierantoni CR, Costa JDO, Stralen ACDSV, Lauar TV, et al. Avaliação do escopo de prática de médicos participantes do Programa Mais Médicos e fatores associados. Ciênc Saúde Colet 2016; 21:273948.

43. Xierali IM. Physician multisite practicing: impact on access to care. J Am Board Fam Med 2018; 31:260-9.

44. Holmes GM, Fraher EP. Developing physician migration estimates for workforce models. Health Serv Res 2017; 52:529-45.
45. Holmes GM, Morrison M, Pathman DE, Fraher E. The contribution of "plasticity" to modeling how a community's need for health care services can be met by different configurations of physicians. Acad Med 2013; 88:1877-82.

46. Viana ALd'A, Miranda AS, Silva HP. Segmentos institucionais de gestão em saúde: descrição, tendências e cenários prospectivos. In: Noronha JC, Lima LD, Chorny AH, Dal Poz MR, Gadelha P, organizadores. Brasil Saúde Amanhã: dimensões para o planejamento da atenção à saúde. Rio de Janeiro: Editora Fiocruz; 2015. p. 151-88.

47. Miranda AS. Institucionalidades jurídicas e administrativas de estabelecimentos de saúde nas regiões do Brasil. (Novos Caminhos, 16). http://www.resbr.net.br/wp-content/ uploads/2017/04/Novos_Caminhos_16.pdf.

48. Coelho VSP. Contratação de serviços e regio nalização. (Novos Caminhos, 13). http://www. resbr.net.br/wp-content/uploads/2017/02/ Novos-Caminhos-13.pdf. 


\section{Abstract}

The article aims to analyze physicians' commuting from a new perspective, verifying their movement and work supply between health regions, specifically in five regions in the state of São Paulo, Brazil. This movement was referred to as physicians commuting, defined as the diversity of job situations over the course of a given time period in given geographic territories. The methodology used was a multiple case study with quantitative and qualitative approaches. All the physicians registered in the National Registry of Healthcare Establishment (CNES) in March 2015 were categorized as either "exclusive physicians", with employment contracts exclusively in the target health region or "nonexclusive physicians", with employment contracts both in that region and in other regions. We analyzed the region's socioeconomic and health characteristics and healthcare structure. The region's dependence on external physicians, namely those residing in other regions, varied from 30 to 40\%; dependence was higher in the more economically developed regions and less in the less developed regions. Internal dependence, among municipalities, was close to $40 \%$ in the regions with higher economic development and reached 60\% in the less developed regions. Non-exclusive physicians tended to be more specialized, working more in surgical and diagnostic specialties, while exclusive physicians worked more in basic and clinical specialties, suggesting that the commuting patterns are associated with the organization of different arrangements of healthcare provision. We identified a growing share of outsourced arrangements and the importance of regionally negotiated actions. Such studies can better orient more integrated redistributive policies.

Regional Health Planning; Health Manpower; Physicians; Internal Migration; Health Systems

\section{Resumen}

El objetivo de este artículo es analizar el movimiento de los médicos, desde una nueva perspectiva, verificando su desplazamiento y oferta de trabajo entre regiones de salud, específicamente, en cinco regiones del estado de São Paulo, Brasil. Se denominó este movimiento circularidad médica, y se definió por la diversidad de los vínculos que se constituyen durante el ejercicio profesional, observado a lo largo de un determinado período, en determinados espacios geográficos. La metodología utilizada fue la del estudio de casos múltiples con aplicación de abordajes cuantitativos y cualitativos. Todos los médicos registrados en la base del Registro Nacional de Establecimientos (CNES), en marzo de 2015, se categorizaron como: "médicos exclusivos", con vínculos exclusivamente en la región en cuestión; $y$ "médicos no exclusivos", con vínculos en esta región y en otras. Se analizaron los datos socioeconómicos y de salud de la región y la estructura asistencial de salud. La dependencia regional de médicos externos varió de un 30 a un 40\%, fue más elevada en las regiones más desarrolladas y menor en las menos desarrolladas. La dependencia interna, entre municipios, es cercana al $40 \%$ en las regiones con mayor desarrollo económico, y llega a un 60\% en las regiones menos desarrolladas. Los médicos no exclusivos están más especializados, con una mayor actuación en especialidades quirúrgicas y de diagnóstico, mientras que los exclusivos actúan más en especialidades básicas y clínicas, indicando que el movimiento puede estar asociado a la organización de la prestación de la asistencia, en sus diferentes configuraciones. Se identifica una creciente participación de soluciones tercerizadas, así como la importancia de las acciones consensuadas regionalmente. Este tipo de estudios pueden orientar mejor las políticas redistributivas e integrarlas más.

Regionalización; Recursos Humanos en Salud; Médicos; Migración Interna; Sistemas de Salud

Recebido em 09/Jul/2018

Versão final reapresentada em 26/Jan/2019

Aprovado em 28/Fev/2019 